\title{
Genomic and human papillomavirus profiling of an oral cancer cohort identifies TP53 as a predictor of overall survival
}

\author{
Neil Mundi ${ }^{1,2^{*}}$ D, Stephenie D. Prokopec ${ }^{3}$, Farhad Ghasemi ${ }^{1}$, Andrew Warner ${ }^{4}$, Krupal Patel ${ }^{1}$, Danielle MacNeil ${ }^{1,4}$, \\ Christopher Howlett ${ }^{5}$, William Stecho ${ }^{5}$, Paul Plantinga ${ }^{5}$, Nicole Pinto ${ }^{1,4}$, Kara M. Ruicci ${ }^{1,4}$, Mohammed Imran Khan ${ }^{1}$, \\ Myung Woul Han ${ }^{6}$, John Yoo ${ }^{1,4}$, Kevin Fung ${ }^{1,4}$, Axel Sahovaler ${ }^{1}$, David A. Palma ${ }^{4}$, Eric Winquist ${ }^{4}$, Joe S. Mymryk ${ }^{1,4,7}$, \\ John W. Barrett ${ }^{1,4}$, Paul C. Boutros ${ }^{3,8,9+}$ and Anthony C. Nichols ${ }^{1,4 \dagger}$
}

\begin{abstract}
Abtract
Background: The genomic landscape of head and neck cancer has been reported through The Cancer Genome Atlas project. We attempt to determine if high-risk human papillomavirus (HPV) or frequently mutated genes are correlated with survival in an oral cancer cohort.
\end{abstract}

Methods: Patient demographic data along with data from final pathology was collected. Tumor DNA was analyzed using a custom Illumina targeted sequencing panel. Five high-risk HPV types were tested by qPCR. Statistical analyses were used to identify associations between patient outcome and mutational status.

Results: High-risk HPV types were identified in 7\% of cases; HPV status was not associated with survival. Mutations were identified in TP53, TERT promoter, \& PIK3CA. Mutations in TP53 were significantly associated with poorer overall survival on multi-variate analysis $(p=0.03)$.

Conclusions: Mutations in TP53 were associated with poor patient survival. Expanding our sample size may identify further predictors of outcome to direct customized cancer care.

Keywords: Oral cancer, Genomics, Mutational status, tp53, Survival

\section{Introduction}

Oral cavity squamous cell carcinoma (OSCC) is a significant public health problem worldwide, with over 350, 000 new cases diagnosed yearly and greater than 150,000 annual deaths [1]. Unfortunately, both the disease process and treatment modalities can have a profound negative impact on patient quality of life, due to difficulty in eating and speaking, disfigurement and chronic pain. In addition, survival rates for OSCC continue to be poor, with fewer than $50 \%$ of patients with advanced disease living 5 years following diagnosis [2].

\footnotetext{
* Correspondence: neilmundi@gmail.com

${ }^{\dagger}$ Paul C. Boutros and Anthony C. Nichols contributed equally to this work.

${ }^{1}$ Department of Otolaryngology - Head and Neck Surgery, Western University, London, ON, Canada

${ }^{2}$ Victoria Hospital, London Health Science Centre, Room B3-431A, 800

Commissioners Road East, London, ON N6A 5W9, Canada

Full list of author information is available at the end of the article
}

Exposure to tobacco and alcohol represent conventional risk factors for the development of OSCC. However, oral human papillomavirus (HPV) infection has recently been discovered to be an additional risk factor for a significant number of head and neck cancers, particularly those arising in the oropharynx. The most common subtypes identified by The Cancer Genome Atlas (TCGA) were HPV16 (84\%), HPV33 (11\%), HPV35 (4\%) and HPV56 (1\%) [3]. Importantly, these HPV related oropharyngeal cancers experience markedly improved survival relative to HPV negative tumors [4-6]. HPV related cancers contribute to a small percentage of OSCC [7], however the prognostic importance of HPV infection in this site remains unclear [8-10].

The large sample size (515 patients) and the robust, high quality tumor molecular data from TCGA head and neck cancer cohort delivers a rich opportunity for biomarker discovery. In particular, genetic changes such 
as TP53 mutations and 3p arm deletion events were previously found to be predictors of tumor recurrence and overall patient survival within the TCGA cohort [11]. Importantly, validation of these variants in independent cohorts has been performed, but with varying results due to differences in disease site, treatment rendered and base study population [12-15]. Here, we attempt to expand on these findings by evaluating the association of HPV status on patient survival, as well as the impact of mutation status using a list of frequently mutated genes discovered by TCGA, using a prospectively collected cohort of 135 OSCCs.

\section{Materials and methods}

\section{Patient population}

Ethics approval was obtained from the University of Western Ontario Research Ethics Board (REB 16579). One hundred and thirty-six patients with OSCC treated at the London Regional Cancer Program at the London Health Sciences Centre with primary surgery were prospectively consented and enrolled between 2011 and 2015 (note that 1 patient was later excluded due to poor sequencing quality). Patient primary tumors, matched blood and clinical data was collected, including age at diagnosis, use of tobacco and alcohol, 7th edition American Joint Committee on Cancer (AJCC) TNM stage, treatment regimen, and post-treatment follow up information.

\section{Sample collection and DNA extraction}

Fresh tumor was harvested from the center of the ablation specimen after the resection was complete, with care taken not to disturb the surgical margins. Tumor cellularity of $>70 \%$ was confirmed by frozen section analysis. Ten $\mathrm{mL}$ of whole blood was obtained by venipuncture or arterial line during the anesthetic for the majority of patients $(118 / 136,87 \%)$. The tumor was placed on ice and transported to the research laboratory, where a portion was frozen and another piece underwent immediate DNA extraction using Qiagen kits (Cat \#: 80204). DNA was similarly extracted from blood samples, also using Qiagen kits (Cat \#: 51104).

\section{Library generation and targeted sequencing of oral cavity samples}

Nine genes were selected for targeted sequencing based on results from the TCGA Head and Neck Squamous Cell Carcinoma (HNSCC) cohort, including CDKN2A, NOTCH1, PIK3CA, TP53, FAT1, CASP8, COL11A1, and $H R A S$, as well as the TERT promoter region. A custom capture for these nine genes was designed using the Illumina Ampliseq platform. Tumor and matched blood samples (118 patients with matched blood, 22 tumor alone) were processed at the London Regional Genomics Centre on the Illumina MiSeq platform. FASTQ files were downloaded and aligned to GRCh38 using BWAMEM (v0.7.15). Duplicates were not marked (due to the highly targeted nature of the data). Indel realignment and recalibration was performed using GATK (v3.7.0). A minimum of $80 \mathrm{x}$ (tumor) or $50 \mathrm{x}$ (normal) coverage across at least $80 \%$ of target sequences was obtained in $78 \%$ of the samples (201/257; Additional file 1: Figure S1). A single tumor had very poor coverage and was removed from downstream analyses (Additional file 2: Figure S2). Similarly, a single normal sample also had very poor coverage and was removed. The matched tumor sample was subsequently treated as tumor only. For tumors with a matched normal, germline single nucleotide polymorphisms were called using GATKs HaplotypeCaller (v3.7.0) and filtered for quality and read depth. Somatic single nucleotide variants (SNVs) were identified using MuTect (v1.1.7); for tumors without a matched normal sample, a panel of normals (PoN) [generated using 438 normal samples from the TCGA HNSC dataset (BWA v0.7.12 with hs37d5, GATK v3.4.0, MuTect v1.1.6, converted to GRCh38 coordinates using picard v2.7.1)] was used to remove probable germline variants (along with the following filters: SNV passed quality control, had a minimum read depth of 50x and was present in fewer than 4 samples in the PoN). Somatic SNVs were filtered to remove non-functional, off-target (intronic/intergenic) variants prior to downstream analyses. Statistical analyses were performed in the $\mathrm{R}$ statistical environment (v3.4.3), with visualizations generated using the BPG (v5.9.8) with the lattice (v0.20-38) and latticeExtra (v0.6-28) packages.

\section{Determination of HPV status in oral cavity patients}

TaqMan primers and probes that were described previously $[6,16,17]$ were used to determine the HPV status of the oral cavity tumor samples for HPV types 16, 18, 33,35 and 56.

\section{Statistical analyses}

Descriptive statistics were generated for baseline patient, tumor and treatment characteristics for all patients that had successfully sequenced samples $(n=135)$. Univariate and multivariate Cox Proportional Hazards Regression was performed to identify significant $(p<0.05)$ predictors of overall survival (OS) and disease-free survival (DFS). Multivariate analysis was performed by first constructing cox models of OS and DFS with all appropriate variables (as identified by univariate analyses), with a backwards stepwise approach to attain the best fitting models of survival. Kaplan-Meier estimates were generated for OS and DFS for all patients. All statistical analyses were performed in SAS (v9.4; SAS institute, Cary $\mathrm{NC}$ ) using two-sided statistical testing at the 0.05 significance level. 


\section{Results}

Baseline tumor, patient and treatment characteristics Patient demographics and clinical characteristics are summarized in Table 1 . The majority of patients were male, with a mean age at diagnosis of 62.2 years. Patients had a mean smoking history of 26.2 pack years and $40.6 \%$ of patients consumed more than twenty-one alcoholic drinks per week. Five percent of patients had positive margins, however an additional 39\% of cases had close margins (defined as $<3 \mathrm{~mm}$ at our institution). Adjuvant radiotherapy alone was provided to $45 \%$ of patients $(61 / 135)$, while $26 \%$ of patients $(35 / 135)$ received adjuvant chemoradiation (Table 1). The average follow-up for patients was 32 months.

\section{Mutational landscape and tumor HPV status}

Ten tumors (7\%) were found to be HPV positive. Of these, nine had evidence of HPV-16 and one carried HPV-33. No tumors were found to harbor HPV types 18,35 or 56 . Gene-wise mutation status for patient tumors and their characteristics are displayed in Fig. 1. A total of 52 unique functional variants were detected in TP53, with mutations observed in $42 \%$ of patients. Of these, 5 patients each harbored mutations affecting splice sites while 7 harbored nonsense mutations (with $5 / 7$ occurring within exon 6 , potentially truncating the DNA-binding domain, Additional file 4: Table S1). The remainder had missense mutations, of which $60 \%$ were within the DNA binding domain that are thought to have the greatest impact on function and prognosis [13,

Table 1 Patient Demographics

\begin{tabular}{lll}
\hline Variable & Value & Number of Patients \\
\hline HPV & Negative & 123 \\
Gender & Positive & 12 \\
& Male & 97 \\
Age & Female & 38 \\
Smoking & Mean =62.3 SD =11.1 & \\
& Heavy (> 20 py) & 71 \\
Alcohol Abuse & Light (<= 20 py) & 26 \\
& Never (0 py) & 38 \\
T stage & Yes & 54 \\
& No & 81 \\
N stage & T0-T2 & 57 \\
& T3-T4 & 78 \\
Adjuvant Therapy & No-N2a & 89 \\
& N2b-N3 & 46 \\
& None & 39 \\
& Radiation & 61 \\
& Chemoradiation & 35 \\
\hline
\end{tabular}

18]. TP53 mutations were more frequent in HPVnegative tumors (chi-squared proportions test $p=0.029$ ). For the remaining targeted genes, FAT1, NOTCH1, CDKN2A, PIK3CA, COL1A11, and CASP8, mutations were identified in $25,20,18,18,12$, and $9 \%$ of patients respectively (Fig. 1). Unfortunately, the capture for HRAS failed for unknown reasons and it was excluded from downstream analyses. The TERT promoter was mutated in $30.4 \%$ of the samples (Fig. 1), with common variants $\mathrm{C} 228 \mathrm{~T}$ and $\mathrm{C} 250 \mathrm{~T}$ observed in 62 and $31 \%$ of patients respectively. These appeared to be mutually exclusive. An additional variant, TERT C228A, appeared in 3 patients. Clinical variables were assessed for associations with variant status of each gene. CASP8 and TERT promoter mutations were significantly more frequent in patients with less than 10 pack years smoking history (chi-squared proportions test, $p<0.01$, Additional file 3: Figure S3). CASP8 mutations were also associated with a younger age at diagnosis (student's t-test, $p=0.009$ ).

\section{Mutations within TP53 are associated with poor overall survival}

Univariate analysis revealed $\mathrm{T}$ and $\mathrm{N}$ stage and the use of adjuvant chemotherapy were predictors of poorer overall survival $(p<0.05$, Additional file 5: Table S2). Female gender, advanced $\mathrm{N}$ stage and use of adjuvant chemotherapy were associated with poorer disease-free survival ( $p<0.05$, Additional file 6: Table S3). HPV status and mutations in any gene were not found to be predictive of OS or DFS in univariate analysis (Additional file 5: Table S2 and Additional file 6: Table S3). This was also the case when restricting analysis to mutations within the p53 DNA binding domain. However, known predictors of outcome were significantly associated with OS, including $\mathrm{T}$ and $\mathrm{N}$ stage (Additional file 5: Table S2 and Additional file 6: Table S3). Backward stepwise multivariate analysis resulted in a model for OS that included age, HPV status, $\mathrm{T}$ and $\mathrm{N}$ stage and TP53 mutation status (Table 2). In this model, TP53 mutations were associated with poorer $\mathrm{OS}(\mathrm{HR}=1.96$ [1.06-3.60], $p=0.03$; Table 2, Fig. 2).

\section{Discussion}

Biomarker studies are frequently hampered by low sample numbers with poor statistical power, the lack of reproducibility of the assay, the heterogeneity of the treatment delivered and the limitations of retrospectively collected data [18, 19]. Here, we performed genomic characterization and HPV typing of a large, prospectively collected cohort of oral cavity cancers treated according to National Comprehensive Cancer Network guidelines, paired with high quality Illumina sequencing, rigorous bioinformatics quality metrics and HPV testing methods that have been thoroughly validated by our group $[6,16$, 


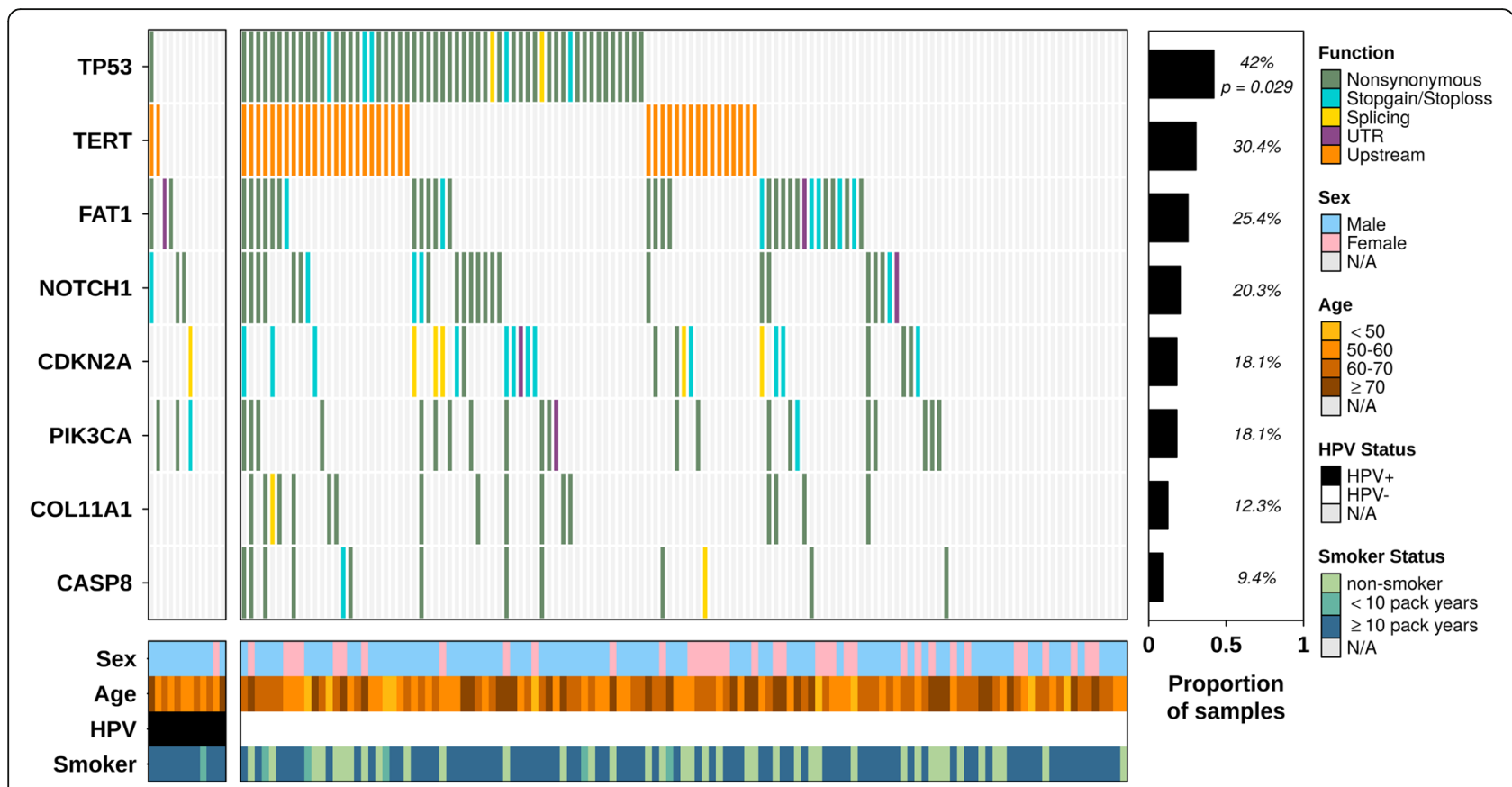

Fig. 1 Mutation and HPV status of oral cancer cohort. SNV profile for each gene/patient stratified by HPV-positive and negative cases (on left and right, respectively). Barplot indicates recurrence (proportion of cohort) while covariates show patient sex, age, HPV status and smoking status. TP53 mutations were enriched in HPV-negative cohort $(p=0.029)$

17, 20]. Importantly, matched normal DNA was available for the majority of cases $(87 \%)$ to serve as a reference control, which our group has recently demonstrated is critical to generating highly accurate mutation calls (Sun et al., under revision at Nature Methods). Thus, with high quality sequencing, matched DNA, prospectively collected tumors and clinical data, we aimed to generate a robust cohort to examine molecular features able to predict patient outcome. We found that the mutational landscape and frequency of HPV-positive disease (7\%) was similar to existing OSCC literature [7, 21, 22]. Su et al. demonstrated a mutation frequency of $42.5 \%$ in 136 oral cavity squamous cell tumors, while Poeta et al. sequenced 420 patients with HNSCC and found the TP53 mutation frequency in oral cavity samples to be $53 \%$ which are similar to our mutation frequency of $42 \%$ $[23,24]$. However, the mutation frequency we observed was lower than that of the TCGA analysis and the investigation by Pickering et al. [21, 22]. This heterogeneity of results may be the result of a number of factors

Table 2 Multivariate Analysis of Overall and Disease-Free Survival

\begin{tabular}{|c|c|c|c|c|}
\hline \multicolumn{5}{|l|}{ Overall Survival } \\
\hline Variable & Comparison & Hazard Ratio & $95 \% \mathrm{Cl}$ & HR p-value \\
\hline Age & & 1.02 & $0.993-1.05$ & 0.148 \\
\hline T stage & T3-T4 vs. T0-T2 & 1.84 & $0.960-3.53$ & 0.0661 \\
\hline N stage & N2b-N3 vs. NO-N2a & 4.63 & $2.43-8.82$ & $3.16 \mathrm{E}-06$ \\
\hline HPV & Positive vs. negative & 2.42 & $0.817-7.19$ & 0.1107 \\
\hline TP53 & Mutant vs. wildtype & 1.96 & $1.06-3.60$ & 0.0311 \\
\hline \multicolumn{5}{|l|}{ Disease-Free Survival } \\
\hline Variable & Comparison & Hazard Ratio & $95 \% \mathrm{Cl}$ & HR p-value \\
\hline Gender & Male vs. Female & 2.98 & $1.27-7.01$ & 0.0122 \\
\hline N stage & N2b-N3 vs. N0-N2a & 3.12 & $1.52-6.40$ & 0.00188 \\
\hline HPV & Positive vs Negative & 2.25 & $0.847-5.96$ & 0.104 \\
\hline Adjuvant Chemotherapy & Yes vs. No & 1.77 & $0.885-3.52$ & 0.107 \\
\hline
\end{tabular}




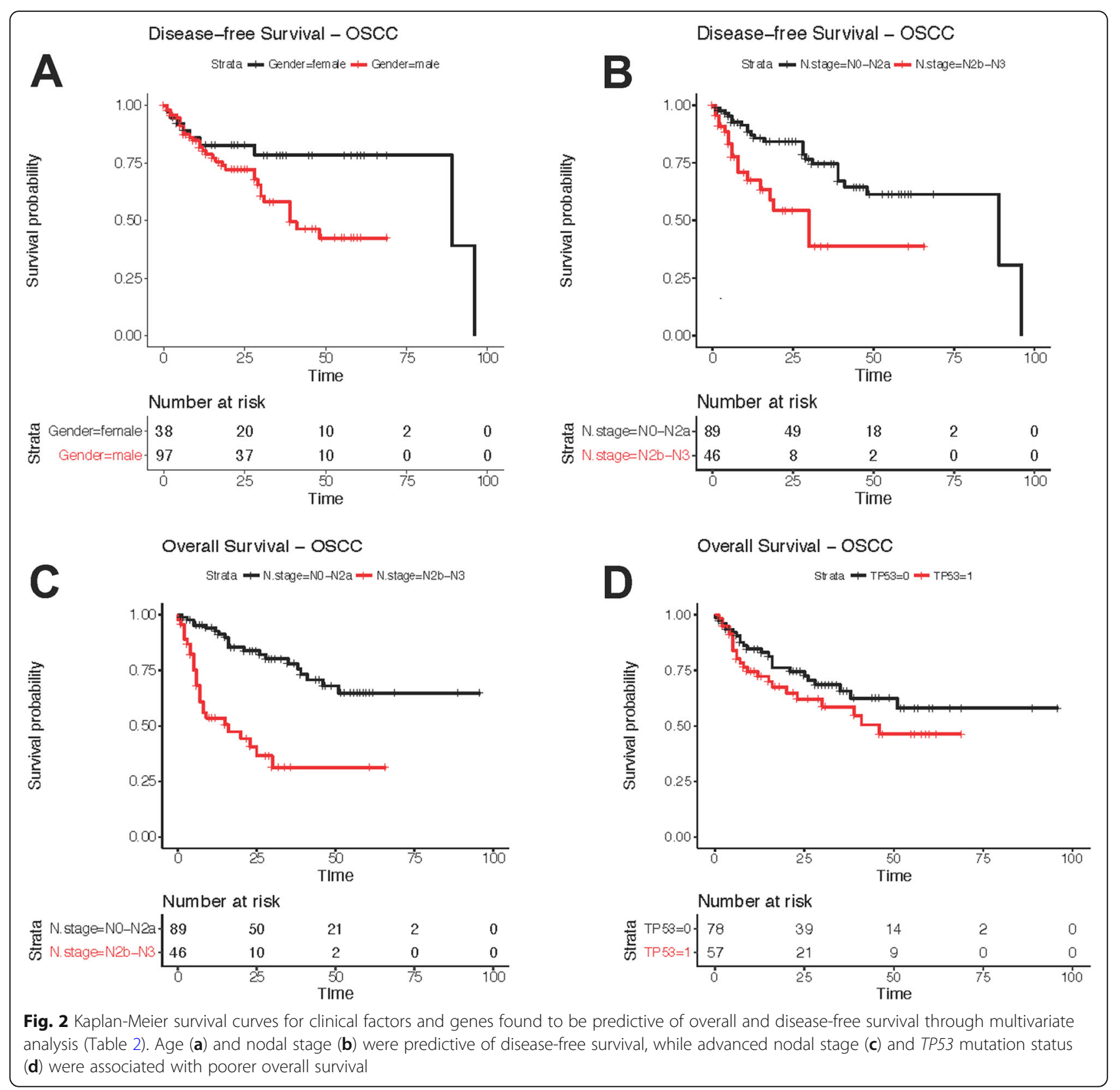

including the large number of institutes contributing to the TCGA study versus our biobank collection derived from a single geographic region. In addition, the fact that our analysis is limited to only the oral cavity may also contribute to the differences mentioned. No genes were found to predict survival on their own; however, in multivariate analysis TP53 mutations were associated with poorer OS, consistent with other reports [11, 13-15, 25].

The p53 protein encoded by TP53 is a transcription factor, which is often referred to as the "cellular gatekeeper" due to its role in protecting cellular integrity by directing the cell's physiological response to insults such as DNA damage and hypoxia. TP53 is the most frequently mutated gene in human cancers $(\sim 50-60 \%$ of cancers) [19]. In head and neck cancer, over $80 \%$ of HPV-negative tumors harbor TP53 mutations, compared to 3\% of HPV-positive tumors [21]. In agreement with this, we observed that all but one of the TP53 mutations occurred in the HPVnegative cohort. It was previously suggested that because the HPV-oncoprotein E6 leads to degradation of p53, selective pressure for loss of function mutations in this gene are unnecessary [26].

Previous analyses of TCGA's HNSCC cohort identified TP53 mutations as a predictor of poorer overall survival, 
with a hazard ratio (HR) of $2.8 \pm 0.8$ [11]. This was particularly true for missense mutations occurring within the 190 codons of the DNA-binding domain of P53, as well as for splicing and non-sense mutations [8]. However, even mutations that were not anticipated to impact P53 function were found to predict poorer survival compared to wild-type tumors $(2.2 \pm 0.7)$. Neskey and colleagues further examined the OSCCs within TCGA that were treated with primary surgery with or without adjuvant therapy $(n=168)$, as well as a separate validation cohort of 96 OSCC patients [13]. They classified tumors with non-synonymous P53 mutations using a novel evolutionary action (EA) score to stratify patients into low- and high-risk groups [13], revealing P53 to be a significant predictor of patient outcome. We have carefully reviewed this data and attempted to classify our observed mutations using the published website for the EA score: http://mammoth.bcm.tmc.edu/EAp53/. We noted that the score cannot be calculated for nonsense and splicing mutations, which make up a significant portion of our TP53 mutations $(12 / 52,23 \%)$. In addition, the website was not able to calculate a score for some of our missense variants. Thus, we did not include the EA score in our survival analysis. We did not identify any specific mutation groups associated with OS or DFS (Additional file 5: Table S2 and Additional file 6: Table S3). However, P53 mutations, when accounting for other patient and tumor characteristics, were found to be significant for OS (HR = 1.96 [95\%: 1.06-3.60]). However, examination of the survival curves in the aforementioned studies, as well as the fact that P53 mutation status did not also predict DFS in our study, begs the question of whether this marker is accurate enough to guide therapeutic decisions. Certainly, P53 mutation status it is not as strong of a predictive marker as p16 in the oropharynx, which has an HR of 0.28 in the context of prospective randomized trials [4]. Likely, P53 mutation status will have to be paired with other markers with molecular and cellular features of tumors that may offer better predictive markers for therapy to build a more reliable predictive model.

In TCGA's HNSCC cohort, a subset of tumors were enriched for both CASP8 and HRAS mutations [21]. Interestingly, these tumors tend to be P53 wild-type and have a quiescent copy number profile [21]. In vivo, CASP8-mutant xenografts have been found to have significantly higher engraftment rates and tumor burden, and lower survival compared to wild-type xenografts [22], suggesting that tumors carrying these mutations may be more aggressive. However, we observed no correlation between CASP8 mutations and survival, in either TCGA or the current cohort [21]. This may reflect the low frequency of CASP 8 mutations in both datasets, and suggests a much larger sample size may be required to tease out these details.
Tumors carrying activating mutations in the TERT promoter region tend to be more aggressive, both in head and neck and other cancer types [12, 27-31]. Evidence exists to suggest that these mutations are overrepresented in patients failing their first line of treatment [12]. Despite being detected in a high fraction of our cohort (30.4\%), TERT promoter mutations were not associated with OS or DFS. Morris et al. studied a cohort of recurrent and/or metastatic head and neck and cancers and identified TERT promoter mutations exclusively in HPV-negative patients (16/29) with none detected in HPV-positive patients $(n=20)$ [12]. This is consistent with the ability of the HPV oncoprotein E6 to activate telomerase independently [32], potentially precluding the need for an activating mutation in the promoter region. We surprisingly identified TERT promoter mutations in two HPV-positive patients, which we separately confirmed by conventional and real-time $\mathrm{qPCR}$ (data not shown). Further study is needed to understand the role of TERT in both HPV-positive and negative disease.

Despite our concerted efforts to develop a robust biomarker for patient outcome in OSCC, our study faced a number of limitations: the failure of the HRAS capture, lack of matched normal DNA for 22 samples, the small number of genes tested, and a sample size of 135 . These limitations will need to be addressed in future studies to identify effective biomarkers of failure that can be translated to clinical care.

The data and conclusions reported herein represent an addition to a growing collection of evidence of the impact of P53 mutations on survival in head and neck cancer. Sandulache et al. recently reported an association between high-risk TP53 mutations and extra-nodal extension in oral cavity cancer for example [15]. Lapke et al. observed that missense mutations in the TP53 DNA-binding domain were an independent prognostic factor for shorter diseasefree survival [33]. Investigations such as these and our own serve to further validate the findings of the TCGA. In our case, we studied data obtained from patients treated at a single surgical center with a single disease subsite using a streamlined treatment protocol; importantly, this served to control for confounding factors that can influence the fidelity of patient outcome data.

\section{Conclusions}

The mutational landscape and frequency of HPV detection in OSCC was consistent with the literature. Mutations in TP53 were associated with poor overall survival, highlighting this gene as a potential biomarker for prediction of patient outcome. Expanding our sample size and increasing the number of genetic features studied may identify further predictors of outcome to direct customized patient care. 


\section{Supplementary information}

Supplementary information accompanies this paper at https://doi.org/10. 1186/s41199-019-0045-0.

Additional file 1: Figure S1. Following alignment, coverage of target sequences was evaluated for each sample. A minimum of $80 x$ (tumor) or 50x (normal) coverage across at least $80 \%$ of target sequences was obtained in 78\% of samples (201/257).

Additional file 2: Figure S2. A single tumor sample [504] had very poor coverage and was removed from downstream analyses. Similarly, a single normal sample [395] also had very poor coverage and was removed. The matched tumor sample was subsequently treated as tumor only.

Additional file 3: Figure S3. Comparison of mutations by smoking status. CASP8 and TERT promoter mutations occurred more frequently in patients with less than 10 pack year smoking history.

Additional file 4: Table S1. Summary of TP53 mutations.

Additional file 5: Table S2. Univariate Analysis for Overall Survival.

Additional file 6: Table S3. Univariate Analysis for Disease-free Survival.

\section{Abbreviations}

AJCC: American Joint Committee on Cancer; DFS: Disease-Free Survival; HNSCC: Head and Neck Squamous Cell Carcinoma; HPV: Human Papilloma Virus; OS: Overall Survival; OSCC: Oral Squamous Cell Carcinoma; SNV: Single Nucleotide Variation; TCGA: The Cancer Genome Atlas

\section{Acknowledgements}

Not applicable

\section{Authors' contributions}

$A N, N M, J B, P B, J M$ for study concepts. AN, JB, JM, CH for study design. AN, $\mathrm{NM}, \mathrm{AS}, \mathrm{KP}, \mathrm{NP}, \mathrm{KR}, \mathrm{MH}, \mathrm{MK}, \mathrm{CH}$ for data acquisition. $\mathrm{AN}$, JB for quality control of data and algorithms. SP, FG, NM, WS, PP for data analysis and interpretation. SP, FG, AW for statistical analysis. NM, AN, JB for manuscript preparation. DM, KF, JY, EW, KP, DP for manuscript editing. All authors participated in manuscript review. All authors read and approved the final manuscript.

\section{Funding}

Canadian Institute for Health Research MOP 340674.

\section{Availability of data and materials}

Please contact author for data requests.

\section{Ethics approval and consent to participate}

University of Western Ontario REB 16579. Consent for participation is not applicable as our investigation does not report on individual data.

\section{Consent for publication}

Not applicable

\section{Competing interests}

The authors declare that they have no competing interests.

\section{Author details}

${ }^{1}$ Department of Otolaryngology - Head and Neck Surgery, Western University, London, ON, Canada. ${ }^{2}$ Victoria Hospital, London Health Science Centre, Room B3-431A, 800 Commissioners Road East, London, ON N6A 5W9, Canada. ${ }^{3}$ Ontario Institute for Cancer Research, Toronto, ON, Canada. ${ }^{4}$ Department of Oncology, Western University, London, ON, Canada. ${ }^{5}$ Department of Pathology, Western University, London, ON, Canada. ${ }^{6}$ Department of Otolaryngology, College of Medicine, Ulsan University Hospital, University of Ulsan, Ulsan, South Korea. ${ }^{7}$ Department of Microbiology \& Immunology, Western University, London, ON, Canada. ${ }^{8}$ Department of Medical Biophysics, University of Toronto, Toronto, ON, Canada. ${ }^{9}$ Department of Pharmacology \& Toxicology, University of Toronto, Toronto, ON, Canada.
Received: 16 September 2019 Accepted: 21 November 2019 Published online: 05 December 2019

\section{References}

1. Warnakulasuriya S. Global epidemiology of oral and oropharyngeal cancer. Oral Oncol. 2009:45(4-5):309-16.

2. Pulte $\mathrm{D}$, Brenner $\mathrm{H}$. Changes in survival in head and neck cancers in the late 20th and early 21st century: a period analysis. Oncologist. 2010; 15(9):994-1001.

3. Bratman SV, et al. Human papillomavirus genotype association with survival in head and neck squamous cell carcinoma. JAMA Oncol. 2016:2(6):823-6.

4. Ang KK, et al. Human papillomavirus and survival of patients with oropharyngeal cancer. N Engl J Med. 2010;363(1):24-35.

5. Chaturvedi AK, et al. Human papillomavirus and rising oropharyngeal cancer incidence in the United States. J Clin Oncol. 2011:29(32):4294-301.

6. Nichols AC, et al. The epidemic of human papillomavirus and oropharyngeal cancer in a Canadian population. Curr Oncol. 2013; 20(4):212-9.

7. Lingen MW, et al. Low etiologic fraction for high-risk human papillomavirus in oral cavity squamous cell carcinomas. Oral Oncol. 2013;49(1):1-8.

8. Burr AR, et al. HPV impacts survival of stage IVC non-oropharyngeal HNSCC cancer patients. Otorhinolaryngol Head Neck Surg. 2018;3(1).

9. Ko HC, et al. Prognostic implications of human papillomavirus status for patients with non-oropharyngeal head and neck squamous cell carcinomas. J Cancer Res Clin Oncol. 2017;143(11):2341-50.

10. Salazar $C R$, et al. Human papillomavirus-associated head and neck squamous cell carcinoma survival: a comparison by tumor site and initial treatment. Head Neck Pathol. 2014;8(1):77-87.

11. Gross AM, et al. Multi-tiered genomic analysis of head and neck cancer ties TP53 mutation to 3p loss. Nat Genet. 2014;46(9):939-43.

12. Morris LG, et al. The Molecular Landscape of Recurrent and Metastatic Head and Neck Cancers: Insights From a Precision Oncology Sequencing Platform. JAMA Oncol. 2016:3(2):244-55.

13. Neskey DM, et al. Evolutionary action score of TP53 identifies high-risk mutations associated with decreased survival and increased distant metastases in head and neck Cancer. Cancer Res. 2015;75(7):1527-36.

14. Osman AA, et al. Evolutionary action score of TP53 coding variants is predictive of platinum response in head and neck Cancer patients. Cancer Res. 2015;75(7):1205-15.

15. Sandulache VC, et al. High-risk TP53 mutations are associated with Extranodal extension in Oral cavity squamous cell carcinoma. Clin Cancer Res. 2018;24(7):1727-33.

16. Nichols AC, et al. Does HPV type affect outcome in oropharyngeal cancer? J Otolaryngol Head Neck Surg. 2013:42:9.

17. Nichols AC, et al. High frequency of activating PIK3CA mutations in human papillomavirus-positive oropharyngeal cancer. JAMA Otolaryngol Head Neck Surg. 2013;139(6):617-22.

18. Vargas AJ, Harris CC. Biomarker development in the precision medicine era: lung cancer as a case study. Nat Rev Cancer. 2016;16(8):525-37.

19. Ludwig JA, Weinstein JN. Biomarkers in cancer staging, prognosis and treatment selection. Nat Rev Cancer. 2005:5(11):845-56.

20. Um SH, et al. Variable expression of the forgotten oncogene E5 in HPVpositive oropharyngeal cancer. J Clin Virol. 2014;61(1):94-100.

21. Cancer Genome Atlas, N. Comprehensive genomic characterization of head and neck squamous cell carcinomas. Nature. 2015:517(7536):576-82.

22. Pickering $C R$, et al. Integrative genomic characterization of oral squamous cell carcinoma identifies frequent somatic drivers. Cancer Discov. 2013;3(7):770-81.

23. Poeta ML, et al. TP53 mutations and survival in squamous-cell carcinoma of the head and neck. N Engl J Med. 2007;357(25):2552-61.

24. Su SC, et al. Exome sequencing of Oral squamous cell carcinoma reveals molecular subgroups and novel therapeutic opportunities. Theranostics. 2017;7(5):1088-99.

25. Ma J, et al. Mutation allele frequency threshold does not affect prognostic analysis using next-generation sequencing in oral squamous cell carcinoma. BMC Cancer. 2018:18(1):758.

26. Ruttkay-Nedecky B, et al. Relevance of infection with human papillomavirus: the role of the p53 tumor suppressor protein and E6/E7 zinc finger proteins (review). Int J Oncol. 2013;43(6):1754-62.

27. Jung $\mathrm{SJ}$, et al. Mutation of the TERT promoter leads to poor prognosis of patients with non-small cell lung cancer. Oncol Lett. 2017;14(2):1609-14. 
28. Vinothkumar $\mathrm{V}$, et al. TERT promoter hot spot mutations are frequent in Indian cervical and oral squamous cell carcinomas. Tumour Biol. 2016; 37(6):7907-13.

29. Chau NG, et al. Incorporation of next-generation sequencing into routine clinical care to direct treatment of head and neck squamous cell carcinoma. Clin Cancer Res. 2016;22(12):2939-49.

30. Qu Y, et al. TERT promoter mutations predict worse survival in laryngeal cancer patients. Int J Cancer. 2014;135(4):1008-10.

31. Cancer Genome Atlas Research, N. Integrated genomic characterization of papillary thyroid carcinoma. Cell. 2014;159(3):676-90.

32. Liu X, et al. HPV E6 protein interacts physically and functionally with the cellular telomerase complex. Proc Natl Acad Sci U S A. 2009;106(44): 18780-5.

33. Lapke N, et al. Missense mutations in the TP53 DNA-binding domain predict outcomes in patients with advanced oral cavity squamous cell carcinoma. Oncotarget. 2016;7(28):44194-210.

\section{Publisher's Note}

Springer Nature remains neutral with regard to jurisdictional claims in published maps and institutional affiliations.

Ready to submit your research? Choose BMC and benefit from:

- fast, convenient online submission

- thorough peer review by experienced researchers in your field

- rapid publication on acceptance

- support for research data, including large and complex data types

- gold Open Access which fosters wider collaboration and increased citations

- maximum visibility for your research: over $100 \mathrm{M}$ website views per year

At BMC, research is always in progress.

Learn more biomedcentral.com/submissions 